\title{
Calculation of Electron-Induced X-ray Intensities under Grazing Exit Conditions *
}

\author{
Kouichi Tsuji ${ }^{\dagger}$ and Kouhei Tetsuoka \\ Department of Applied Chemistry, Graduate School of Engineering, \\ Osaka City University, Sugimoto 3-3-138, Sumiyoshi-ku Osaka 558-8585 Japan \\ Filip Delalieux \\ Department of Chemistry, University of Antwerp Universiteitsplein 1, B-2610 Antwerp, Belgium \\ Shigeo Sato \\ Nissan Arc Co. 1 Natsushima-Cho, Yokosuka, 237-0061, Japan \\ (Received 3 October 2003; Accepted 4 November 2003; Published 18 December 2003)
}

\begin{abstract}
Electron probe microanalysis (EPMA) under grazing-exit conditions allows us to perform microscopic surface analysis, thin-film analysis and particle analysis. To evaluate the thickness and density of thin-films, it is necessary to calculate X-ray intensities as a function of the exit angle. In this paper, we propose a procedure to calculate X-ray intensities as a function of grazing-exit angle. Monte Carlo simulation is used to determine the depth distribution of the electron-induced X-ray production and a multilayer model is applied to include the grazing emission process. The exit angle resolved curves, calculated for Ni thin films, were compared with experimental curves. A good agreement between the experimental plots and the calculated curves indicates that the proposed computational approach can be applied successfully to the calculation of EPMA intensities. Appling this calculation, the lateral resolution of EPMA was also evaluated at different take-off angles. It was suggested that the lateral resolution would be improved under grazing-exit conditions. [DOI: 10.1380/ejssnt.2003.111]
\end{abstract}

Keywords: Electron probe microanalysis (EPMA); Monte Carlo simulation; Grazing exit; Depth distribution; Energy dispersive X-ray (EDX) detector; $\mathrm{Ni}$

\section{INTRODUCTION}

The penetration depth of electrons in solid samples strongly depends on the energy of the electrons. It is possible to perform surface and thin-film analysis by electron probe microanalysis (EPMA) using low energy electrons [1-3]. The study of low energy EPMA is gaining interest in the field of X-ray spectrometry. However, when we use energy-dispersive X-ray detectors with a low energy resolution, the selection of analytical lines becomes serious problem in some cases.

A recently developed method, 'grazing-exit EPMA (GE-EPMA)', enables surface-sensitive analysis. GEEPMA has successfully been applied to localized thin-film analysis and single-particle analysis [4-6]. Compared to conventional EPMA, the most distinctive characteristic of GE-EPMA is in control of the take-off angle for detection of characteristic X-rays. In the case of GE-EPMA, $\mathrm{X}$-rays are measured at grazing angles smaller than 1 degree. This special configuration makes surface sensitive analysis possible. X-rays, which are produced deep inside a sample, are strongly absorbed in the sample before they reach the detector. In addition, these X-rays are refracted at the surface of the sample, and are obstructed by slit, which is fixed in front of the detector. Therefore, these Xrays are not detected through the slit under grazing-exit conditions.

Thin-film analysis is one of the useful applications of GE-EPMA. In principle, by analogy to grazing exit Xray fluorescence [7], it is possible to estimate the thick-

\footnotetext{
* This paper was presented at The 4th International Symposium on Atomic Level Characterizations for New Materials and Devices (ALC '03), Kauai, Hawaii, USA, 5-10 October, 2003.

†Corresponding author: tsuji@a-chem.eng.osaka-cu.ac.jp
}

ness and density of thin films with GE-EPMA [6]. This thin-film analysis is performed by comparing take-off angle resolved X-ray intensities (experimental results) with calculated angle dependent curves. Actually, the authors have performed thin-film analysis using this curve fitting method $[7,8]$. The characteristic X-ray intensities were calculated with a multilayer model $[6,7]$. In this calculation, however, the actual depth-distribution of the electron induced X-ray production was not taken into account. It was assumed that the X-rays were produced homogeneously throughout the thin film. Only the grazing exit process was considered for the calculation. However, strictly speaking, the depth distribution of the Xray production should be included in the calculation of the X-ray intensities at grazing exit angles. In this paper, we propose a computational approach to calculate GEEPMA intensities, which combines two phenomena: the depth distribution of X-ray production and the exit angle dependence of emitted X-rays.

\section{CALCULATIONS}

\section{A. Depth Distribution of Generated X-rays}

In a solid sample, electrons are scattered in various directions. To simulate the trajectories of these electrons, Monte Carlo simulation can be used [9]. Multiple- elastic scattering and inelastic scattering can be included in the simulations. As a result, depth and lateral distributions of electrons and characteristic X-rays can be obtained. It has been shown that the obtained results agree well with the experimental results of EPMA [9-11].

Monte Carlo simulation software packages are widely available. The CASINO program [12-14] is one of them, which was developed by research team of R. Gauvin (Sher- 


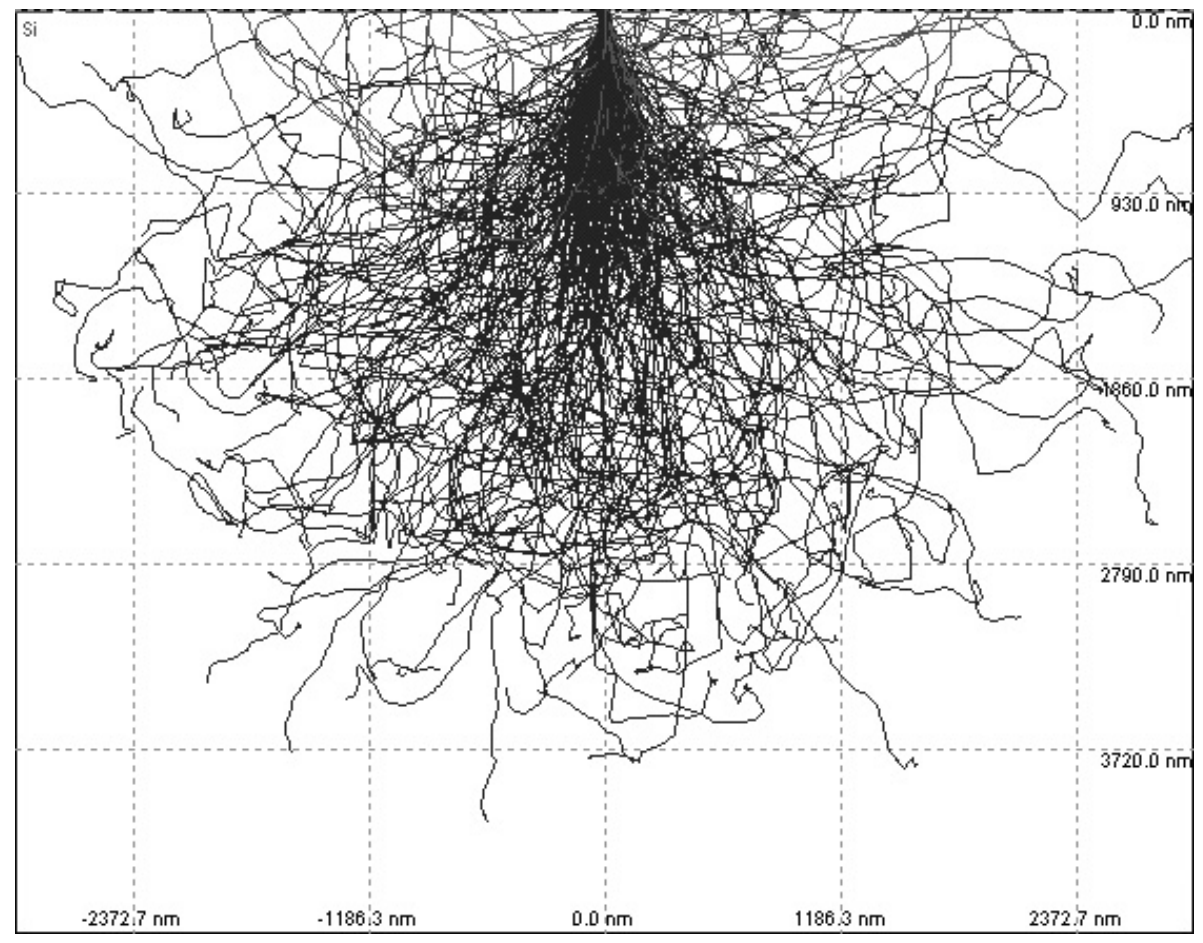

FIG. 1: Typical electron trajectories simulated with CASINO. Material: Si, number of electron trajectories calculated: 100,000 (number displayed: 200), beam diameter: $50 \mathrm{~nm}$, electron energy: $20 \mathrm{keV}$.

\section{Si Ko Intensity (PRZ)}

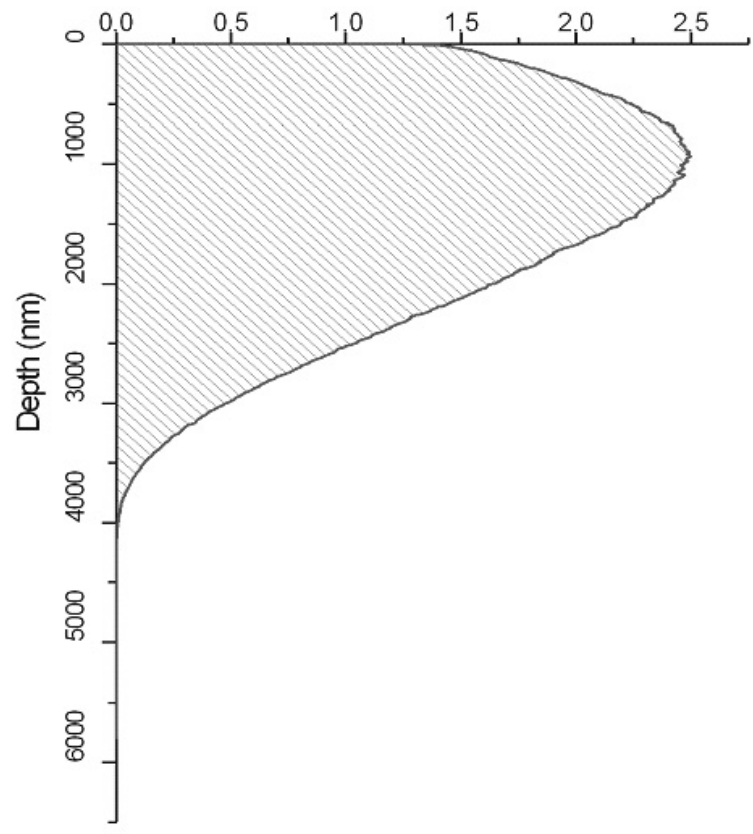

FIG. 2: Depth distribution of Si K $\alpha$ X-rays induced by electrons (energy: $20 \mathrm{keV}$, number: 100,000) in a Si wafer.

brooke University, Sherbrooke, Canada). In CASINO simulations, many important parameters, such as sample composition, electron energies, number of electrons, incident angle of the electrons, etc. can easily be changed. Figure 1 shows a typical example of electron trajectories, simulated with CASINO. Although a total of 100,000 electrons were used in the actual simulations, Fig. 1 only shows the trajectories of 200 electrons not to obscure the picture. Finally, the depth $(Z)$ distribution $\left(I_{e}(Z)\right)$ of the $\mathrm{X}$-ray intensity generated by electrons was obtained. Figure 2 shows a typical distribution of $\mathrm{Si}_{\alpha}$ induced by electrons (20 keV, 100,000 electrons) as a function of the depth in the Si wafer.

\section{B. X-ray Emission at Grazing Angles}

Next, we had to take into account the X-ray emission process at grazing exit angles. At these angles, refraction and reflection on the surface and other material interfaces are important phenomena. As a general approximation, we have used a multilayer model, which is shown in Fig.3. Details of the calculations are described elsewhere [7]. In this model, we assume that X-rays, having the same energies as the analyzed X-rays, are impinging on the sample surface at a grazing incident angle. According to the reciprocity theorem $[15,16]$, the intensities of the $\mathrm{X}$-rays, which are emitted at a depth $\mathrm{Z}$ in the $j$ th layer and which are observed at a grazing exit angle, are proportional to the X-ray intensities, which are impinging at the same grazing incident angle and which are produced at the depth $\mathrm{Z}$ in the $j$ th layer. These $\mathrm{X}$-rays produce an electric field $\left(E_{j}(Z)\right)$ at a depth $Z$ in the $j$ th layer as shown in Fig. 3. Therefore, the X-ray intensities $\left(I_{j}\right)$ emitted from the $j$ th layer can be expressed as follows:

$$
I_{j} \propto \int_{0}^{d_{j}}\left|E_{j}(Z)\right|^{2} d Z
$$



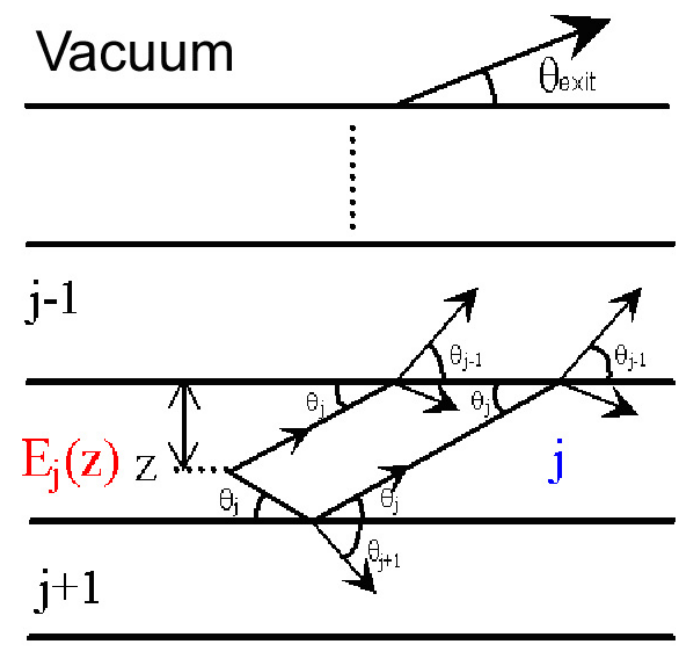

$\vdots$

FIG. 3: Multilayer model used to calculate X-ray intensities emitted at grazing angles.

By using Eq. (1), it is possible to calculate the intensities of characteristic X-rays as a function of the exit angle. Equation (1) can be applied to various samples, such as a flat substance with a single surface layer or multiple surface layers.

\section{Calculation of Electron-Induced X-ray Intensities at Grazing Exit Angles}

The intensities of electron-induced X-rays observed at grazing exit angles can be calculated by combining the two processes described previously: i.e., calculation of the depth distribution of the X-ray production and calculation of X-ray emissions at grazing exit angles. Thus, the equation consists of the terms of $I_{e}(Z)$ and $E(Z)$ as follows:

$$
I_{j} \propto \int_{0}^{d_{j}} I_{e}(Z)\left|E_{j}(Z)\right|^{2} d Z
$$

The absorption effect of the characteristic X-rays in each layer is included in this equation.

\section{RESULTS AND DISCUSSIONS}

\section{A. Thin-Film Analysis}

Details of the GE-EPMA setup used in this study have been described elsewhere $[6,17]$. To change the angle of detection, the energy dispersive X-ray (EDX) detector was moved vertically using a linear positioning $(Z-)$ stage. A slit of $0.2 \mathrm{~mm}$ was fixed at a distance of about $100 \mathrm{~mm}$ from the sample in front of the EDX detector. A computer controlled both the EDX analysis and the $Z$-stage.

Using Eq. (2), the intensities of $\mathrm{Ni} \mathrm{K} \alpha \mathrm{X}$-rays emitted from a single $\mathrm{Ni}$ layer on a $\mathrm{Si}$ wafer were calculated as a

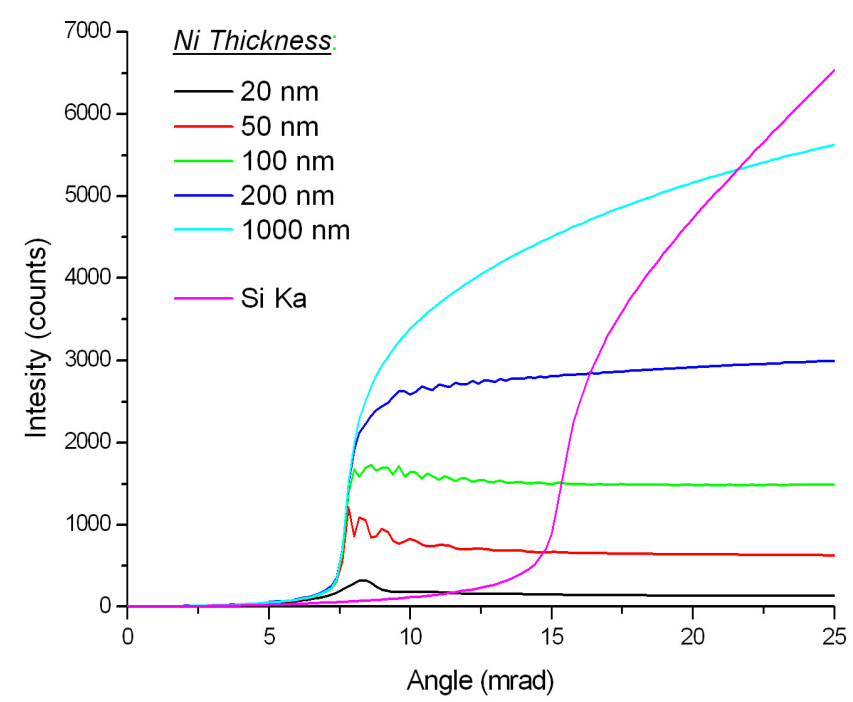

FIG. 4: Calculated angle-dependences of $\mathrm{Ni} \mathrm{K} \alpha$ intensities emitted from $\mathrm{Ni}$ thin layers (with different thicknesses) on a Si substrate.

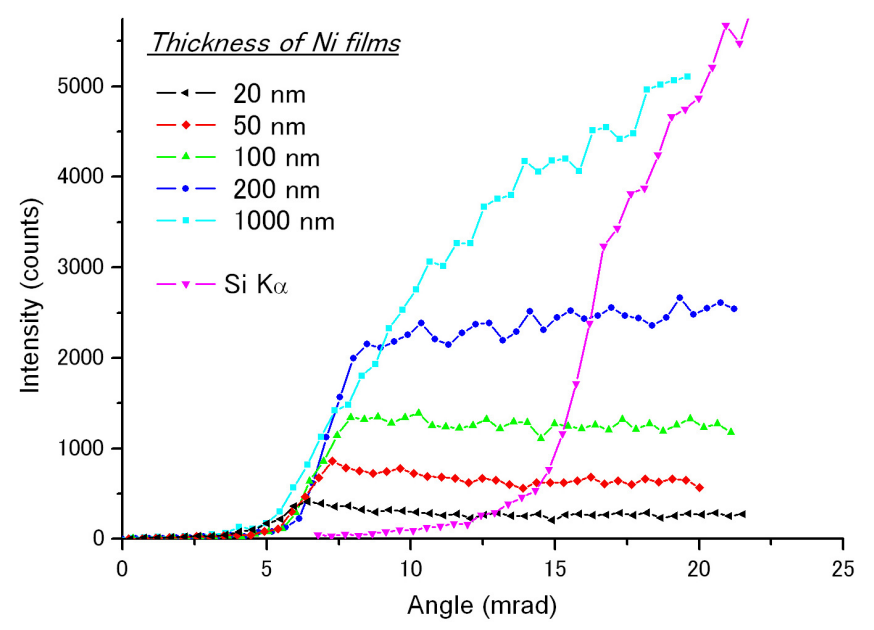

FIG. 5: Experimentally obtained plots for Ni K $\alpha$ intensities emitted from $\mathrm{Ni}$ thin layers (with different thicknesses) on a Si substrate.

function of exit angle. The numerical results for different thicknesses of Ni layers are shown in Fig. 4. It is evident that the shape of the angle-dependent curves changes depending on the thickness. Oscillation structures can also be observed, which are caused by the interference between the reflected X-rays and refracted X-rays in the layer. The period of the oscillations depends on the thickness of this layer. Therefore, in principle, the thickness of the thin film can be evaluated from the shape of the curve and the period of the oscillations.

The experimental results are given in Fig. 5. The $\mathrm{Ni}$ thin films were prepared on $\mathrm{Si}$ wafers by magnetron sputtering. The thickness was controlled by adjusting the sputtering time after measuring the sputtering rate. For these samples, the intensities of $\mathrm{Ni} \mathrm{K} \alpha \mathrm{X}$-rays were measured as a function of the exit angle. The experimental curves shown in Fig. 5 agree well with the theoretical 
TABLE I: Comparison of the theoretical lateral resolutions (nm) of conventional EPMA and GE-EPMA, obtained by using Monte Carlo simulations on the interaction of an electron beam (diameter $50 \mathrm{~nm}$ ), with $\mathrm{Si}$, $\mathrm{Cu}$ and Au substrates for different accelerating voltages $(5,10$ and $20 \mathrm{kV})$.

\begin{tabular}{crccc}
\hline \hline Elements & Methods & $5 \mathrm{kV}$ & $10 \mathrm{kV}$ & 899 \\
\hline $\mathrm{Si}$ & Conv. EPMA & 311 & 122 & 1415 \\
& GE-EPMA & 68 & 294 & 171 \\
$\mathrm{Cu}$ & Conv. EPMA & 124 & 121 & 828 \\
& GE-EPMA & 84 & 184 & 148 \\
$\mathrm{Au}$ & Conv. EPMA & 85 & 112 & 421 \\
& GE-EPMA & 62 & 190 \\
\hline \hline
\end{tabular}

\section{Conventional EPMA}

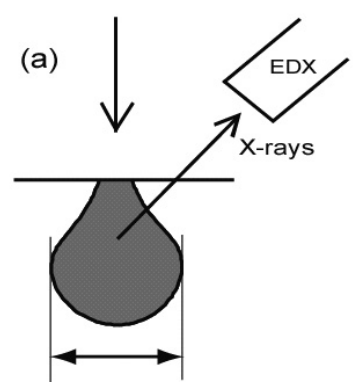

GE-EPMA

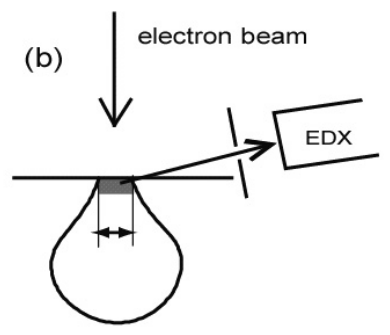

FIG. 6: Schematic representation of the origin of the observed $\mathrm{X}$-rays in EPMA and GE-EPMA respectively, indicating the differences in the cross-sectional dimensions.

curves shown in Fig. 4. The effect of angular divergence due to the slit width was not included in the calculations of the angle-dependence of the Ni $\mathrm{K} \alpha$ intensities. This is probably one of the reasons why in the experimental curves the $\mathrm{Ni} \mathrm{K} \alpha$ intensities do not increase sharply at the critical angle, i.e. near $7.5 \mathrm{mrad}$. In addition, the statistic errors on the $\mathrm{Ni} \mathrm{K} \alpha \mathrm{X}$-ray intensities were too large to observe the oscillations in the angle-dependent curves. To observe these oscillations, it would be necessary to use an electron beam with a higher current, a more efficient X-ray detector system and higher exit angle resolving power, allowing a higher resolution of the experimentally determined angle-dependence.

\section{B. Improving the Lateral Resolution of EPMA}

Due to electron scattering in the sample, characteristic $\mathrm{X}$-rays are produced within an interaction volume that has a significantly larger cross-section than the impinging beam size, as shown in Fig. 6 (a). At conventional detection angles (typically $\sim 40^{\circ}$ for EPMA) the lateral resolution is determined by the dimensions of this interaction volume. In the case of GE-EPMA, however, only the X-rays that are emitted close to the surface (few nm) are detected, as shown in Fig. 6 (b). Therefore, the lateral resolution of GE-EPMA is limited only by the crosssection of the most top layer of the interaction volume. By using Monte Carlo simulations, the spatial distributions of the X-ray production inside different sample materi-

als were evaluated for different electron beam accelerating voltages. As can be seen in Table I, the lateral dimensions of the observed part of the X-ray production volume are reduced considerably under grazing exit conditions, especially for light elements and at large accelerating voltages. For example, when using a $20 \mathrm{keV}$ electron beam (beam diameter: $50 \mathrm{~nm}$ ) the lateral resolution of a conventional EPMA of $\mathrm{Si} \mathrm{K} \alpha$ from a $\mathrm{Si}$ substrate was estimated to be $1415 \mathrm{~nm}$, while that of GE-EPMA was only $171 \mathrm{~nm}$. These results indicate that it is possible to improve the lateral resolution of EPMA by applying grazing exit x-ray measurement.

\section{CONCLUSIONS}

An algorithm was developed to calculate electroninduced X-ray intensities at grazing-exit angles. This computational approach combines two calculation processes: the calculation of the depth distribution of electron-induced X-ray production, and the calculation of X-ray intensities emitted at grazing angles. The calculated angle-dependences were compared with experimentally determined curves, which were obtained for Ni thin films. The calculated values well agree with the experimental results. In principle, it is possible to evaluate the thickness and density of thin films by fitting calculated curves to experimental plots, because the thickness and the density are important parameters that influence both the shape of the curve and the critical angle at which the X-ray intensities increase sharply. Analysis of the oscillation structures that are theoretically expected will be useful to perform more detailed analysis of thin films. However, for this purpose, observed X-ray intensities have to be enhanced considerably. The development of more efficient X-ray detection systems will also be an important task.

\section{Acknowledgments}

One of the authors (Filip Delalieux) was supported by the Japan Society for the Promotion of Science (JSPS). Part of this work was financially supported by the grantin-aid of the JSPS. The authors acknowledge Prof. ChulUn Ro for his useful advices. 
[1] V. D. Scott and G. Love, J. Anal. At. Spectrom. 14, 367 (1999).

[2] E. Boys, Microscopy and Micoranalysis 6, 307 (2002).

[3] I. Barkwshire, P. Karduck, W. P. Rehbach, and S. Richter, Mikrochim Acta 132, 113 (2000).

[4] K. Tsuji, K. Wagatsuma, R. Nullens, and R. E. Van Grieken, Anal. Chem. 71, 2497 (1999).

[5] K. Tsuji, K. Wagatsuma, R. Nullens and R. E. Van Grieken, J. Anal. At. Spectrom. 14, 1711 (1999).

[6] K. Tsuji, K. Saito, K. Asami, K. Wagatsuma, F. Delalieux, and Z. Spolnik, Spectrochim. Acta, Part B 57, 897 (2002).

[7] K. Tsuji and K. Hirokawa. J. Appl. Phys.75, 7189 (1994).

[8] S. Sato, K. Tsuji and K. Hirokawa, Appl. Phys. A 62, 87 (1996).

[9] V. D. Scott, G. Love and S. J. B. Reed, Quantitative Electron-Probe Microanalysis, pp. 212-238, (Ellis Horwood, London, 1995).
[10] J. A. Small, D. E. Newbury, and J. T. Armstrong, Handbook of X-ray Spectrometry, 2nd ed. Eds. R. E Van Grieken and A. A. Markowicz, pp. 811-931 (Marcel Dekker, New York, 2002).

[11] C. H. Ro, K. Y. Oh, J. Osan, J. de Hoog, A. Worobiec, and R. E. Van Grieken, Anal. Chem. 73, 4574 (2001).

[12] CASINO program (http://www.gel.usherb.ca/casino/)

[13] P. Hovington, D. Drouin and R. Gauvin, Scanning, 19, 1 (1997).

[14] P. Hovington, D. Drouin, R. Gauvin, D. C. Joy, and N. Evans, Scanning, 19, 29 (1997).

[15] M. Born and E. Wolf, Principles of Optics, p. 381 (Pergamon, Oxford, 1991).

[16] R. S. Becker, J. A. Golovchenko, and J. R. Patel, Phys. Rev. Lett. 50, 153 (1983).

[17] K. Tsuji, Z. Spolnik and T. Ashino, Rev. Sci. Instrum. 72, 3933 (2001). 